\title{
Sampled-data state estimation for neural networks of neutral type
}

\author{
Changchun Yang ${ }^{1}$, Yongqing Yang ${ }^{1,2^{*}}$, Manfeng $\mathrm{Hu}^{1}$ and Xianyun $\mathrm{Xu}^{1}$
}

\author{
"Correspondence: \\ yyq640613@gmail.com \\ 'School of Science, Jiangnan \\ University, Wuxi, 214122, China \\ ${ }^{2}$ Key Laboratory of Advanced \\ Process Control for Light Industry \\ (Ministry of Education), Jiangnan \\ University, Wuxi, 214122, China
}

\begin{abstract}
In this paper, the sampled-data state estimation is investigated for a class of neural networks of neutral type. By employing a suitable Lyapunov functional, a delay-dependent criterion is established to guarantee the existence of the sampled-data estimator. The estimator gain matrix can be obtained by solving linear matrix inequalities (LMIs). A numerical example is given to show the effectiveness of the proposed method.
\end{abstract}

Keywords: neural network; sampled-data; state estimation; neutral type; LMIs

\section{Introduction}

Over the past decades, considerable attention has been devoted to the study of artificial neural networks due to their extensive application in signal and image processing, pattern recognition, and combinatorial optimization [1,2]. Numerous models of neural networks such as cellular neural networks, Hopfield-type neural networks, Cohen-Grossberg neural networks, and bidirectional associative memory neural networks have been extensively investigated in the literature [3-7].

In recent years, the state estimation problem has been a hot topic because of its great significance to estimation of the neuron states through available output measurements. Many results have been available. For example, the state estimation problem for delayed neural networks was discussed in [8]. Huang and Feng investigated the state estimation of recurrent neural networks with time-varying delay. A delay-dependent condition was derived to guarantee the existence of a desired state estimator $[9,10]$. By constructing a Lyapunov-Krasovskii functional and using a convex combination technique, the exponential state estimation for Markovian jumping neural networks with time-varying discrete and distributed delays was investigated [11]. The state estimation of discrete-time neural networks and fuzzy neural networks was studied in [12-15]. Recently, the state estimation problem of neural networks of neutral type has motivated a great deal of interest. Park et $a l$. investigated the state estimation of neural networks of neutral type with interval timevarying delays [16-18]. A delay-dependent condition was proposed for neural networks of neutral type with mixed time-varying delays and Markovian jumping parameters [19].

At the same time, the sampled-data state estimation of neural networks has gradually caused researchers' concern with the development of computer hardware technology. For instance, $\mathrm{Hu}$ investigated the sampled-data state estimation of delayed neural networks with Markovian jumping parameters [20], and in [21] the sampled-data state estimator was

\section{空 Springer}

(c)2014 Yang et al.; licensee Springer. This is an Open Access article distributed under the terms of the Creative Commons Attribution License (http://creativecommons.org/licenses/by/2.0), which permits unrestricted use, distribution, and reproduction in any medium, provided the original work is properly cited. 
designed for Markovian jumping fuzzy cellular neural networks with mode-dependent probabilistic time-varying delays. In order to effectively deal with the sampled-data, the author investigated the sampled-data state estimation of neural networks by using a discontinuous Lyapunov functional approach $[22,23]$. It is worth mentioning that the approach to estimate the neuron states through the output sampled measurement needs less information from the network outputs, which can lead to a significant reduction of the information communication burden in the network. To the best of our knowledge, the sampled-data state estimation of neural networks of neutral type has not been investigated so far.

Motivated by the above discussion, in this paper, we aim to deal with the sampled-data state estimation problem for neural networks of neutral type. By utilizing Lyapunov functional, Jensen's inequalities and Schur complement, a delay-dependent criterion is given to guarantee the existence of the state estimator of neural networks of neutral type. The estimator gain matrix can be obtained in terms of LMIs.

The organization of the rest is as follows. In Section 2, the problem is formulated and some lemmas are introduced. In Section 3, some sufficient conditions are given to guarantee the existence of the sampled-data estimator. An example is given to exemplify the usefulness of our theoretical results in Section 4. And in the last section, Section 5, we give some conclusions.

\section{Problem formulation}

In this paper, the sampled-data state estimation problem will be investigated. Consider the following neural networks of neural type:

$$
\begin{aligned}
& \dot{x}(t)=-A x(t)+W_{0} f(x(t))+W_{1} g(x(t-h(t)))+V \dot{x}(t-d(t))+J, \\
& y(t)=C x(t),
\end{aligned}
$$

where $x(t)=\left[x_{1}(t), \ldots, x_{n}(t)\right]^{T} \in \mathbb{R}^{n}$ is the neuron state vector associated with $n$ neurons. $f(x(t))=\left[f_{1}\left(x_{1}(t)\right), \ldots, f_{n}\left(x_{n}(t)\right)\right]^{T} \in \mathbb{R}^{n}$ and $g(x(t-h(t)))=\left[g_{1}\left(x_{1}(t-h(t))\right), \ldots, g_{n}\left(x_{n}(t-\right.\right.$ $h(t)))]^{T} \in \mathbb{R}^{n}$ are the neuron activation functions. $J=\left[J_{1}, \ldots, J_{n}\right]^{T}$ is the external input vector at time $t . y(t) \in \mathbb{R}^{m}$ is the measurement output. $A=\operatorname{diag}\left(a_{1}, a_{2}, \ldots, a_{n}\right)$ is a positive diagonal matrix. $W_{0}, W_{1}$, and $V$ are the interconnection matrices representing the weight coefficients of the neurons. $C \in \mathbb{R}^{m \times n}$ is a known constant matrix. $h(t)>0$ and $d(t)>0$ are axonal signal transmission delays that satisfy the following inequalities:

$$
\begin{array}{ll}
0<h(t) \leq \bar{h}, & \dot{h}(t) \leq h_{D}, \\
0<d(t) \leq \bar{d}, & \dot{d}(t) \leq d_{D}<1 .
\end{array}
$$

Assumption 1 The neuron activation functions $f(\cdot), g(\cdot)$ satisfy the Lipschitz condition

$$
\begin{aligned}
& \left\|f\left(x_{1}\right)-f\left(x_{2}\right)\right\| \leq\left\|F\left(x_{1}-x_{2}\right)\right\|, \\
& \left\|g\left(x_{1}\right)-g\left(x_{2}\right)\right\| \leq\left\|G\left(x_{1}-x_{2}\right)\right\|,
\end{aligned}
$$

where $F \in \mathbb{R}^{n \times n}$ and $G \in \mathbb{R}^{n \times n}$ are the known constant matrices. 
The aim of this paper is to present an efficient estimation algorithm to observe the neuron states from the available network sampling output. To this end, the following full order observer is proposed:

$$
\begin{aligned}
& \dot{\hat{x}}(t)=-A \hat{x}+W_{0} f(\hat{x}(t))+W_{1} g(\hat{x}(t-h(t)))+V \dot{\hat{x}}(t-d(t))+J+u(t), \\
& \hat{y}(t)=C \hat{x}(t)
\end{aligned}
$$

where $\hat{x}(t) \in \mathbb{R}^{n}$ is the estimation of neuron state $x(t) . \hat{y}(t) \in \mathbb{R}^{m}$ is the estimated output vector. $u(t) \in \mathbb{R}^{n}$ is the control input, which is assumed to use sampled data as follows:

$$
\begin{aligned}
u(t) & =K\left[y\left(t_{k}\right)-\hat{y}\left(t_{k}\right)\right] \\
& =K C\left[x\left(t_{k}\right)-\hat{x}\left(t_{k}\right)\right], \quad k=0,1,2, \ldots,
\end{aligned}
$$

where $t_{k}$ denotes the sampling instant and satisfies $\lim _{k \rightarrow \infty} t_{k}=\infty$.

Assumption 2 For $k \geq 0$, there exists a known positive constant $\bar{\tau}$ such that the sampling instant $t_{k}$ satisfies $t_{k+1}-t_{k} \leq \bar{\tau}$.

Define the error vector to be $e(t)=x(t)-\hat{x}(t)$, and

$$
\begin{gathered}
\phi(t)=f(x(t))-f(\hat{x}(t)), \\
\varphi(t)=g(x(t))-g(\hat{x}(t)) .
\end{gathered}
$$

Let $\tau(t)=t-t_{k}, t_{k} \leq t<t_{k+1}$, the controller (3) can be represented as follows:

$$
\begin{aligned}
u(t) & =K C e\left(t_{k}\right) \\
& =K C e(t-\tau(t)), \quad t_{k} \leq t<t_{k+1} .
\end{aligned}
$$

Therefore, the error dynamical system can be expressed by

$$
\dot{e}(t)=-A e(t)-K C e(t-\tau(t))+V \dot{e}(t-d(t))+W_{0} \phi(t)+W_{1} \varphi(t-h(t)),
$$

where $\tau(t)$ satisfies $0 \leq \tau(t) \leq \bar{\tau}$.

The following lemmas will be used in deriving the main results.

Lemma 1 ([19], Jensen's inequality) For any constant matrix $Q \in \mathbb{R}^{n \times n}, Q=Q^{T}>0$, scalar $b>0$, and vector function $x:[0, b] \rightarrow \mathbb{R}^{n}$, one has

$$
-\int_{0}^{b} x^{T}(s) Q x(s) d s \leq-\frac{1}{b}\left[\int_{0}^{b} x(s) d s\right]^{T} Q\left[\int_{0}^{b} x(s) d s\right] .
$$

Lemma 2 ([20], Schur complement) Given constant matrices $\Omega_{1}, \Omega_{2}$, and $\Omega_{3}$, where $\Omega_{1}=$ $\Omega_{1}^{T}$ and $\Omega_{2}>0$, then

$$
\Omega_{1}+\Omega_{3}^{T} \Omega_{2}^{-1} \Omega_{3}<0
$$


if and only if

$$
\left[\begin{array}{cc}
\Omega_{1} & \Omega_{3}^{T} \\
\Omega_{3} & -\Omega_{2}
\end{array}\right]<0
$$

\section{Main results}

In this section, we derive a new delay-dependent LMI criterion for the existence of state estimator (2) for neural networks (1).

Theorem 1 For given matrices $F, G$, positive scalars $\bar{h}$ and $0<\alpha<1$, the error system (6) is globally asymptotically stable if there exist eight positive definite matrices $P, Q_{i}(i=$ $1,2,3,4,5,6,7)$, two positive scalars $\beta_{1}$ and $\beta_{2}$, and any matrix $X$ satisfying the following LMI:

$$
\left[\begin{array}{cc}
\Lambda_{1} & \Lambda_{3}^{T} \\
* & -\Lambda_{2}
\end{array}\right]<0
$$

where

$$
\begin{aligned}
& \Lambda_{1}=\left[\begin{array}{ccccccccc}
Z_{1} & \frac{1}{\bar{\tau}} Q_{3}-X C & 0 & 0 & 0 & (\alpha \bar{h})^{-1} Q_{2} & P V & P W_{0} & P W_{1} \\
* & -\frac{2}{\bar{\tau}} Q_{3} & \frac{1}{\bar{\tau}} Q_{3} & 0 & 0 & 0 & 0 & 0 & 0 \\
* & * & -\frac{1}{\bar{\tau}} Q_{3} & 0 & 0 & 0 & 0 & 0 & 0 \\
* & * & * & Z_{2} & \bar{h}^{-1} Q_{2} & (\bar{h}-\alpha \bar{h})^{-1} Q_{2} & 0 & 0 & 0 \\
* & * & * & * & Z_{3} & 0 & 0 & 0 & 0 \\
* & * & * & * & * & Z_{4} & 0 & 0 & 0 \\
* & * & * & * & * & * & -\left(1-d_{D}\right) Q_{7} & 0 & 0 \\
* & * & * & * & * & * & * & -\beta_{1} I & 0 \\
* & * & * & * & * & * & * & * & Z_{5}
\end{array}\right], \\
& \Lambda_{3}^{T}=\left[\begin{array}{ccc}
-\bar{h} A^{T} P & -\bar{\tau} A^{T} P & -A^{T} P \\
-\bar{h} C^{T} X^{T} & -\bar{\tau} C^{T} X^{T} & -C^{T} X^{T} \\
0 & 0 & 0 \\
0 & 0 & 0 \\
0 & 0 & 0 \\
0 & 0 & 0 \\
\bar{h} V^{T} P & \bar{\tau} V^{T} P & V^{T} P \\
\bar{h} W_{0}^{T} P & \bar{\tau} W_{0}^{T} P & W_{0}^{T} P \\
\bar{h} W_{1}^{T} P & \bar{\tau} W_{1}^{T} P & W_{1}^{T} P
\end{array}\right] \text {, } \\
& \Lambda_{2}=\left[\begin{array}{ccc}
\bar{h}\left(2 P-Q_{2}\right) & 0 & 0 \\
* & \bar{\tau}\left(2 P-Q_{3}\right) & 0 \\
* & * & 2 P-Q_{7}
\end{array}\right] \text {, } \\
& Z_{1}=-P A-A^{T} P+Q_{1}+Q_{5}+Q_{6}+G^{T} Q_{4} G-\frac{1}{\bar{\tau}} Q_{3}-(\alpha \bar{h})^{-1} Q_{2}+\beta_{1} F^{T} F \text {, } \\
& Z_{2}=-\left(1-h_{D}\right) Q_{5}-(\bar{h}-\alpha \bar{h})^{-1} Q_{2}-\bar{h}^{-1} Q_{2}-\beta_{2} G^{T} G \text {, } \\
& Z_{3}=-Q_{6}-\bar{h}^{-1} Q_{2}, \quad Z_{5}=-\left(1-h_{D}\right) Q_{4}-\beta_{2} I, \\
& Z_{4}=-\left(1-\alpha h_{D}\right) Q_{1}-(\bar{h}-\alpha \bar{h})^{-1} Q_{2}-(\alpha \bar{h})^{-1} Q_{2},
\end{aligned}
$$

and then the estimator gain can be designed as $K=P^{-1} X$. 
Proof Consider the following Lyapunov functional:

$$
V=\sum_{i=1}^{8} V_{i},
$$

where

$$
\begin{aligned}
& V_{1}=e^{T}(t) P e(t), \quad V_{2}=\int_{t-\alpha h(t)}^{t} e^{T}(s) Q_{1} e(s) d s, \\
& V_{3}=\int_{t-\bar{h}}^{t} \int_{s}^{t} \dot{e}^{T}(\theta) Q_{2} \dot{e}(\theta) d \theta d s, \quad V_{4}=\int_{-\bar{\tau}}^{0} \int_{t+\theta}^{t} \dot{e}^{T}(s) Q_{3} \dot{e}(s) d s d \theta, \\
& V_{5}=\int_{t-h(t)}^{t} \varphi^{T}(s) Q_{4} \varphi(s) d s, \quad V_{6}=\int_{t-h(t)}^{t} e^{T}(s) Q_{5} e(s) d s, \\
& V_{7}=\int_{t-\bar{h}}^{t} e^{T}(s) Q_{6} e(s) d s, \quad V_{8}=\int_{t-d(t)}^{t} \dot{e}^{T}(s) Q_{7} \dot{e}(s) d s .
\end{aligned}
$$

Calculating the derivative of $V_{i}$ along the trajectories of system (6), we have

$$
\begin{aligned}
\dot{V}_{1} & =2 e^{T}(t) P\left[-A e(t)-K C e(t-\tau(t))+V \dot{e}(t-d(t))+W_{0} \phi(t)+W_{1} \varphi(t-h(t))\right], \\
\dot{V}_{2} & =e^{T}(t) Q_{1} e(t)-(1-\alpha \dot{h}(t)) e^{T}(t-\alpha h(t)) Q_{1} e(t-\alpha h(t)) \\
& \leq e^{T}(t) Q_{1} e(t)-\left(1-\alpha h_{D}\right) e^{T}(t-\alpha h(t)) Q_{1} e(t-\alpha h(t)), \\
\dot{V}_{3} & =\bar{h}^{T} \dot{e}^{T}(t) Q_{2} \dot{e}(t)-\int_{t-\bar{h}}^{t} \dot{e}^{T}(s) Q_{2} \dot{e}(s) d s, \\
\dot{V}_{4} & =\bar{\tau} \dot{e}^{T}(t) Q_{3} \dot{e}(t)-\int_{t-\bar{\tau}}^{t} \dot{e}^{T}(s) Q_{3} \dot{e}(s) d s, \\
\dot{V}_{5} & =\varphi^{T}(t) Q_{4} \varphi(t)-(1-\dot{h}(t)) \varphi^{T}(t-h(t)) Q_{4} \varphi(t-h(t)) \\
& \leq e^{T}(t) G^{T} Q_{4} G e(t)-\left(1-h_{D}\right) \varphi^{T}(t-h(t)) Q_{4} \varphi(t-h(t)), \\
\dot{V}_{6} & =e^{T}(t) Q_{5} e(t)-(1-\dot{h}(t)) e^{T}(t-h(t)) Q_{5} e(t-h(t)) \\
& \leq e^{T}(t) Q_{5} e(t)-\left(1-h_{D}\right) e^{T}(t-h(t)) Q_{5} e(t-h(t)), \\
\dot{V}_{7} & =e^{T}(t) Q_{6} e(t)-e^{T}(t-\bar{h}) Q_{6} e(t-\bar{h}), \\
\dot{V}_{8} & =\dot{e}^{T}(t) Q_{7} \dot{e}(t)-(1-\dot{d}(t)) \dot{e}^{T}(t-d(t)) Q_{7} \dot{e}(t-d(t)) \\
& \leq \dot{e}^{T}(t) Q_{7} \dot{e}(t)-\left(1-d_{D}\right) \dot{e}^{T}(t-d(t)) Q_{7} \dot{e}(t-d(t)) .
\end{aligned}
$$

Note that the Lyapunov functions $-\int_{t-\bar{h}}^{t} \dot{e}^{T}(s) Q_{2} \dot{e}(s) d s$ and $-\int_{t-\bar{\tau}}^{t} \dot{e}^{T}(s) Q_{3} \dot{e}(s) d s$ have the following relationship:

$$
\begin{aligned}
-\int_{t-\bar{h}}^{t} \dot{e}^{T}(s) Q_{2} \dot{e}(s) d s= & -\int_{t-\alpha h(t)}^{t} \dot{e}^{T}(s) Q_{2} \dot{e}(s) d s-\int_{t-h(t)}^{t-\alpha h(t)} \dot{e}^{T}(s) Q_{2} \dot{e}(s) d s \\
& -\int_{t-\bar{h}}^{t-h(t)} \dot{e}^{T}(s) Q_{2} \dot{e}(s) d s, \\
-\int_{t-\bar{\tau}}^{t} \dot{e}^{T}(s) Q_{3} \dot{e}(s) d s= & -\int_{t-\tau(t)}^{t} \dot{e}^{T}(s) Q_{3} \dot{e}(s) d s-\int_{t-\bar{\tau}}^{t-\tau(t)} \dot{e}^{T}(s) Q_{3} \dot{e}(s) d s .
\end{aligned}
$$


By Lemma 1, we have

$$
\begin{aligned}
& -\int_{t-\alpha h(t)}^{t} \dot{e}^{T}(s) Q_{2} \dot{e}(s) d s \leq-(\alpha \bar{h})^{-1}\left[\int_{t-\alpha h(t)}^{t} \dot{e}(s) d s\right]^{T} Q_{2}\left[\int_{t-\alpha h(t)}^{t} \dot{e}(s) d s\right] \\
& \leq-(\alpha \bar{h})^{-1}[e(t)-e(t-\alpha h(t))]^{T} Q_{2}[e(t)-e(t-\alpha h(t))] \\
& =-(\alpha \bar{h})^{-1}\left[e^{T}(t) Q_{2} e(t)-2 e^{T}(t) Q_{2} e(t-\alpha h(t))\right. \\
& \left.+e^{T}(t-\alpha h(t)) Q_{2} e(t-\alpha h(t))\right] \\
& -\int_{t-h(t)}^{t-\alpha h(t)} \dot{e}^{T}(s) Q_{2} \dot{e}(s) d s \leq-(\bar{h}-\alpha \bar{h})^{-1}\left[\int_{t-h(t)}^{t-\alpha h(t)} \dot{e}(s) d s\right]^{T} Q_{2}\left[\int_{t-h(t)}^{t-\alpha h(t)} \dot{e}(s) d s\right] \\
& \leq-(\bar{h}-\alpha \bar{h})^{-1}[e(t-\alpha h(t))-e(t-h(t))]^{T} \\
& \times Q_{2}[e(t-\alpha h(t))-e(t-h(t))] \\
& =-(\bar{h}-\alpha \bar{h})^{-1}\left[e^{T}(t-\alpha h(t)) Q_{2} e(t-\alpha h(t))\right. \\
& -2 e^{T}(t-\alpha h(t)) Q_{2} e(t-h(t)) \\
& \left.+e^{T}(t-h(t)) Q_{2} e(t-h(t))\right], \\
& -\int_{t-\bar{h}}^{t-h(t)} \dot{e}^{T}(s) Q_{2} \dot{e}(s) d s \leq-(\bar{h})^{-1}\left[\int_{t-\bar{h}}^{t-h(t)} \dot{e}(s) d s\right]^{T} Q_{2}\left[\int_{t-\bar{h}}^{t-h(t)} \dot{e}(s) d s\right] \\
& \leq-(\bar{h})^{-1}[e(t-h(t))-e(t-\bar{h})]^{T} \\
& \times Q_{2}[e(t-h(t))-e(t-\bar{h})] \\
& =-(\bar{h})^{-1}\left[e^{T}(t-h(t)) Q_{2} e(t-h(t))\right. \\
& \left.-2 e^{T}(t-h(t)) Q_{2} e(t-\bar{h})+e^{T}(t-\bar{h}) Q_{2} e(t-\bar{h})\right], \\
& -\int_{t-\tau(t)}^{t} \dot{e}^{T}(s) Q_{3} \dot{e}(s) d s \leq-\frac{1}{\tau(t)}\left[\int_{t-\tau(t)}^{t} \dot{e}(s) d s\right]^{T} Q_{3}\left[\int_{t-\tau(t)}^{t} \dot{e}(s) d s\right] \\
& \leq-\frac{1}{\bar{\tau}}[e(t)-e(t-\tau(t))]^{T} Q_{3}[e(t)-e(t-\tau(t))] \\
& =-\frac{1}{\bar{\tau}}\left[e^{T}(t) Q_{3} e(t)-2 e^{T}(t) Q_{3} e(t-\tau(t))\right. \\
& \left.+e^{T}(t-\tau(t)) Q_{3} e(t-\tau(t))\right] \\
& -\int_{t-\bar{\tau}}^{t-\tau(t)} \dot{e}^{T}(s) Q_{3} \dot{e}(s) d s \leq-\frac{1}{\bar{\tau}}[e(t-\tau(t))-e(t-\bar{\tau})]^{T} Q_{3}[e(t-\tau(t))-e(t-\bar{\tau})] \\
& =-\frac{1}{\bar{\tau}}\left[e^{T}(t-\tau(t)) Q_{3} e(t-\tau(t))-2 e^{T}(t-\tau(t)) Q_{3} e(t-\bar{\tau})\right. \\
& \left.+e^{T}(t-\bar{\tau}) Q_{3} e(t-\bar{\tau})\right] \text {. }
\end{aligned}
$$

From Assumption 1 and (4), for positive scalars $\beta_{i}(i=1,2)$, we have the following inequalities:

$$
\begin{aligned}
& \beta_{1}\left[e^{T}(t) F^{T} F e(t)-\phi^{T}(t) \phi(t)\right] \geq 0 \\
& \beta_{2}\left[e^{T}(t-h(t)) G^{T} G e(t-h(t))-\varphi^{T}(t-h(t)) \varphi(t-h(t))\right] \geq 0 .
\end{aligned}
$$


By combining (10)-(23), it is not difficult to deduce that

$$
\begin{aligned}
\dot{V}= & \sum_{i=1}^{8} \dot{V}_{i} \\
\leq & e^{T}(t)\left[-P A-A^{T} P+Q_{1}+Q_{5}+Q_{6}+G^{T} Q_{4} G-\frac{1}{\bar{\tau}} Q_{3}-(\alpha \bar{h})^{-1} Q_{2}\right. \\
& \left.+\beta_{1} F^{T} F\right] e(t)+2 e^{T}(t)\left[-P K C+\frac{1}{\bar{\tau}} Q_{3}\right] e(t-\tau(t)) \\
& -e^{T}(t-\tau(t))\left[\frac{2}{\bar{\tau}} Q_{3}\right] e(t-\tau(t))+2 e^{T}(t)[P V] \dot{e}(t-d(t)) \\
& -\dot{e}^{T}(t-d(t))\left[\left(1-d_{D}\right) Q_{7}\right] \dot{e}(t-d(t))+2 e^{T}(t)\left[P W_{0}\right] \phi(t) \\
& -\phi^{T}(t)\left[\beta_{1} I\right] \phi(t)+2 e^{T}(t)\left[P W_{1}\right] \varphi(t-h(t)) \\
& -\varphi^{T}(t-h(t))\left[\left(1-h_{D}\right) Q_{4}+\beta_{2} I\right] \varphi(t-h(t)) \\
& +2 e^{T}(t)\left[(\alpha \bar{h})^{-1} Q_{2}\right] e(t-\alpha h(t))-e^{T}(t-\alpha h(t))\left[\left(1-\alpha h_{D}\right) Q_{1}\right. \\
& \left.+(\bar{h}-\alpha \bar{h})^{-1} Q_{2}+(\alpha \bar{h})^{-1} Q_{2}\right] e(t-\alpha h(t)) \\
& +2 e^{T}(t-\tau(t))\left[\frac{1}{\bar{\tau}} Q_{3}\right] e(t-\bar{\tau})-e^{T}(t-\bar{\tau})\left[\frac{1}{\bar{\tau}} Q_{3}\right] e(t-\bar{\tau}) \\
& +\dot{e}^{T}(t)\left[\bar{h} Q_{2}+\bar{\tau} Q_{3}+Q_{7}\right] \dot{e}(t) . \\
& +2 e^{T}(t-\alpha h(t))\left[(\bar{h}-\alpha \bar{h})^{-1} Q_{2}\right] e(t-h(t)) \\
& -e^{T}(t-h(t))\left[\left(1-h_{D}\right) Q_{5}+(\bar{h}-\alpha \bar{h})^{-1} Q_{2}+(\bar{h})^{-1} Q_{2}+\beta_{2} G^{T} G\right] e(t-h(t)) \\
& 2 e^{T}(t-h(t))\left[\bar{h}^{-1} Q_{2}\right] e(t-\bar{h})-e^{T}(t-\bar{h})\left[\bar{h}^{-1} Q_{2}+Q_{6}\right] e(t-\bar{h}) \\
& \\
& \\
&
\end{aligned}
$$

Let $\Upsilon=\left[-A-K C 0000 V W_{0} W_{1}\right]$, and $\eta^{T}(t)=\left[e^{T}(t) e^{T}(t-\tau(t)) e^{T}(t-\bar{\tau}) e^{T}(t-h(t)) e^{T}(t-\right.$ $\left.\bar{h}) e^{T}(t-\alpha h(t)) \dot{e}^{T}(t-d(t)) \phi^{T}(t) \varphi^{T}(t-h(t))\right]$. From (6), we have $\dot{e}(t)=\Upsilon \eta(t)$.

Therefore,

$$
\dot{e}^{T}(t)\left[\bar{h} Q_{2}+\bar{\tau} Q_{3}+Q_{7}\right] \dot{e}(t)=\eta^{T}(t)\left[\Upsilon^{T}\left(\bar{h} Q_{2}+\bar{\tau} Q_{3}+Q_{7}\right) \Upsilon\right] \eta(t)
$$

Thus, changing the variable as $X=P K$, equation (24) can be rewritten as

$$
\dot{V} \leq \eta^{T}(t)\left[\Lambda_{1}+\Upsilon^{T}\left(\bar{h} Q_{2}+\bar{\tau} Q_{3}+Q_{7}\right) \Upsilon\right] \eta(t),
$$

where $\Lambda_{1}$ can be seen from Theorem 1 .

In view of the fact $-P Q_{2}^{-1} P \leq-\left(2 P-Q_{2}\right),-P Q_{3}^{-1} P \leq-\left(2 P-Q_{3}\right),-P Q_{7}^{-1} P \leq-\left(2 P-Q_{7}\right)$. From (9), we can obtain the following inequality:

$$
\left[\begin{array}{cc}
\Lambda_{1} & \Lambda_{3}^{T} \\
* & -\Lambda_{6}
\end{array}\right]<0
$$

where

$$
\Lambda_{6}=\left[\begin{array}{ccc}
\bar{h} P^{T} Q_{2}^{-1} P & 0 & 0 \\
* & \bar{\tau} P^{T} Q_{3}^{-1} P & 0 \\
* & * & P^{T} Q_{7}^{-1} P
\end{array}\right] .
$$


Pre- and post-multiplying $\operatorname{diag}\left\{I, I, I, I, I, I, I, I, I, Q_{2} P^{-1}, Q_{3} P^{-1}, Q_{7} P^{-1}\right\}$ and $\operatorname{diag}\{I, I, I, I, I, I$, $\left.I, I, I, P^{-1} Q_{2}, P^{-1} Q_{3}, P^{-1} Q_{7}\right\}$, respectively, we have

$$
\left[\begin{array}{cc}
\Lambda_{1} & \Lambda_{4}^{T} \\
* & -\Lambda_{5}
\end{array}\right]<0,
$$

where

$$
\begin{aligned}
\Lambda_{4}^{T} & =\left[\begin{array}{ccc}
-\bar{h} A^{T} Q_{2} & -\bar{\tau} A^{T} Q_{3} & -A^{T} Q_{7} \\
-\bar{h}(K C)^{T} Q_{2} & -\bar{\tau}(K C)^{T} Q_{3} & -(K C)^{T} Q_{7} \\
0 & 0 & 0 \\
0 & 0 & 0 \\
0 & 0 & 0 \\
0 & 0 & 0 \\
\bar{h} V^{T} Q_{2} & \bar{\tau} V^{T} Q_{3} & V^{T} Q_{7} \\
\bar{h} W_{0}^{T} Q_{2} & \bar{\tau} W_{0}^{T} Q_{3} & W_{0}^{T} Q_{7} \\
\bar{h} W_{1}^{T} Q_{2} & \bar{\tau} W_{1}^{T} Q_{3} & W_{1}^{T} Q_{7}
\end{array}\right], \\
\Lambda_{5} & =\left[\begin{array}{ccc}
\bar{h} Q_{2} & 0 & 0 \\
* & \bar{\tau} Q_{3} & 0 \\
* & * & Q_{7}
\end{array}\right] .
\end{aligned}
$$

By Lemma 2, inequality (28) is equivalent to the inequality $\left[\Lambda_{1}+\Upsilon^{T}\left(\bar{h} Q_{2}+\bar{\tau} Q_{3}+\right.\right.$ $\left.\left.Q_{7}\right) \Upsilon\right]<0$. This implies that the error dynamic (6) is asymptotically stable. This completes the proof.

Remark 1 In the paper, the controller $u(t)=K\left[y\left(t_{k}\right)-\hat{y}\left(t_{k}\right)\right]$ is designed by the sampled data, which needs less information from the network outputs than the controller in [16, 17]. The sampled-data estimation approach can lead to a significant reduction of the information communication burden in the network and save more computing cost.

To this end, the sampled-data estimation problem has been solved and the desired estimator can be designed by Theorem 1 . In the next section, the effectiveness of the developed sampled-data estimation approach for the neural network of neutral type is shown by a numerical example.

\section{Simulation example}

In this section, a numerical example with simulation results is employed to demonstrate the effectiveness of the proposed method.

Example 1 Consider the neural networks of neutral type with the following parameters:

$$
\begin{aligned}
A & =\left[\begin{array}{lll}
3 & 0 & 0 \\
0 & 2 & 0 \\
0 & 0 & 5
\end{array}\right], \quad W_{0}=\left[\begin{array}{ccc}
0.3 & -0.1 & 0 \\
0.1 & 0.4 & -0.2 \\
-0.2 & 0.1 & 0.2
\end{array}\right], \quad W_{1}=\left[\begin{array}{ccc}
0.1 & 1 & 0.2 \\
-0.1 & 0.3 & -0.1 \\
-0.2 & 0.1 & 0.2
\end{array}\right], \\
V & =\left[\begin{array}{ccc}
0.1 & -0.05 & 0.05 \\
0.05 & 0.1 & -0.05 \\
0.05 & 0.1 & 0.1
\end{array}\right], \quad J=\left[\begin{array}{c}
2 \sin (\pi t)+0.03 t^{2} \\
-\sin (4 t)-0.04 t^{2} \\
1.5 \cos (4 t)+0.01 t^{2}
\end{array}\right], \quad C=I,
\end{aligned}
$$




$$
\begin{aligned}
& f(x)=0.5 \tanh (x), \quad g(x(t-h(t)))=0.4 \tanh (x(t-h(t))), \\
& x(0)=\left[\begin{array}{lll}
-1.5 & 1 & -1.5
\end{array}\right]^{T}, \quad h(t)=0.36(1-\sin (t)), \\
& d(t)=0.25(1-\sin (t)), \quad \bar{\tau}=0.05, \quad \alpha=0.5 .
\end{aligned}
$$

From the parameters above, it can be verified that $F=0.5 I, G=0.4 I$. By solving LMI given in Theorem 1 , the feasible solutions are obtained as follows:

$$
\begin{aligned}
& P=\left[\begin{array}{ccc}
22.5358 & -1.1052 & 0.0140 \\
-1.1052 & 32.2373 & 0.6453 \\
0.0140 & 0.6453 & 14.9831
\end{array}\right], \quad Q_{1}=\left[\begin{array}{ccc}
8.4845 & -0.0169 & -0.0747 \\
-0.0169 & 10.5790 & 0.1719 \\
-0.0747 & 0.1719 & 5.6045
\end{array}\right] \text {, } \\
& Q_{2}=\left[\begin{array}{ccc}
15.2407 & -0.6559 & -0.1345 \\
-0.6559 & 19.8115 & 0.2071 \\
-0.1345 & 0.2071 & 11.4000
\end{array}\right], \quad Q_{3}=\left[\begin{array}{ccc}
18.3501 & -0.1527 & 0.1320 \\
-0.1527 & 19.0875 & 0.0926 \\
0.1320 & 0.0926 & 16.9542
\end{array}\right] \text {, } \\
& Q_{4}=\left[\begin{array}{ccc}
11.8617 & 0.2203 & 0.0955 \\
0.2203 & 18.7959 & 1.1519 \\
0.0955 & 1.1519 & 9.2649
\end{array}\right], \quad Q_{5}=\left[\begin{array}{ccc}
6.8482 & -0.0052 & -0.0725 \\
-0.0052 & 9.5406 & 0.1891 \\
-0.0725 & 0.1891 & 3.8875
\end{array}\right] \text {, } \\
& Q_{6}=\left[\begin{array}{ccc}
8.3328 & -0.0359 & -0.0743 \\
-0.0359 & 10.8433 & 0.1788 \\
-0.0743 & 0.1788 & 5.4375
\end{array}\right], \quad Q_{7}=\left[\begin{array}{ccc}
6.1224 & -0.3327 & 0.1054 \\
-0.3327 & 10.1217 & -0.1539 \\
0.1054 & -0.1539 & 4.1545
\end{array}\right] \text {, } \\
& X=\left[\begin{array}{ccc}
-29.6833 & 0.2433 & -0.3916 \\
0.2433 & -15.3925 & 0.9746 \\
-0.3916 & 0.9746 & -53.1328
\end{array}\right], \quad \beta_{1}=18.9090, \quad \beta_{2}=23.3269 \text {. }
\end{aligned}
$$

Thus, the estimator gain matrix $K$ is as follows:

$$
K=P^{-1} X=\left[\begin{array}{ccc}
-1.3190 & -0.0128 & -0.0102 \\
-0.0372 & -0.4796 & 0.1009 \\
-0.0233 & 0.0857 & -3.5505
\end{array}\right]
$$

The simulation results are shown in Figures 1 and 2 . It is easy to find that the responses of the state estimators track to true states very quickly.

Figure 1 The true state $x(t)$ and its estimate $\hat{x}(t)$.

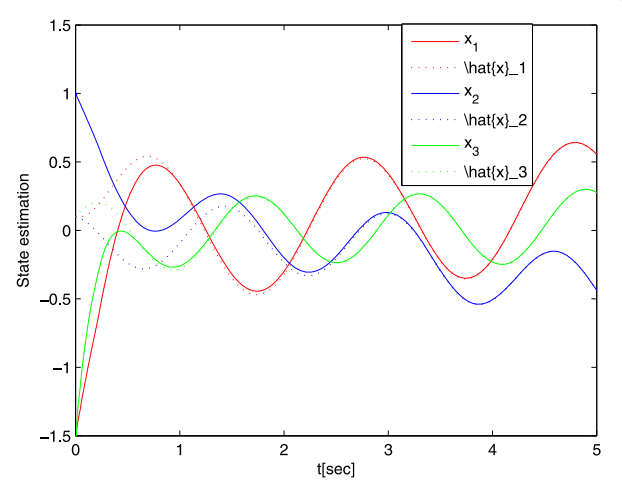


Figure 2 The error of the true state $x(t)$ and its estimate $\hat{x}(t)$.

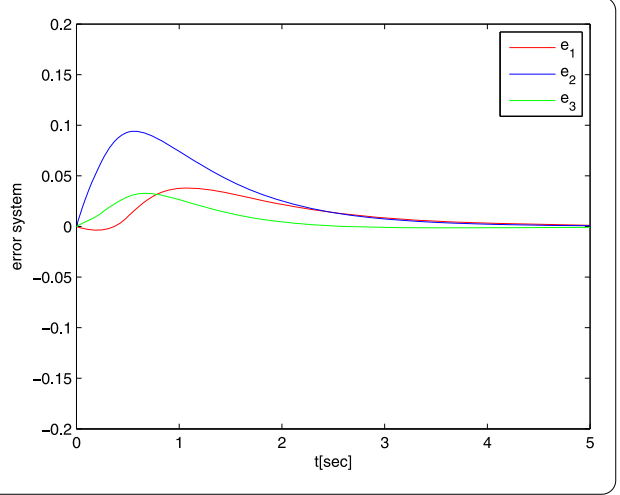

\section{Conclusion}

In this paper, the sampled-data state estimation has been investigated for a class of neural networks of neutral type. By employing a Lyapunov functional, a delay-dependent condition is established to guarantee the existence of the desired state estimator. The feasible solution can be obtained easily by Matlab LMI toolbox. In the end, the numerical example is given to show the effectiveness of the proposed estimator. This work can be extended to the state estimation problem of general systems. This will be done in the near future.

\section{Competing interests}

The authors declare that they have no competing interests.

\section{Authors' contributions}

CY carried out the main results of this paper and drafted the manuscript. YY directed the study and helped to inspect the manuscript. All authors read and approved the final manuscript.

\section{Acknowledgements}

This work was jointly supported by the National Natural Science Foundation of China under Grant 60875036, the Foundation of Key Laboratory of Advanced Process Control for Light Industry (Jiangnan University), Ministry of Education, P.R. China the Fundamental Research Funds for the Central Universities (JUSRP51317B, JUDCF13042).

Received: 18 January 2014 Accepted: 21 April 2014 Published: 09 May 2014

\section{References}

1. Elanayar, V, Yung, C: Radial basis function neural network for approximation and estimation of nonlinear stochastic dynamic systems. IEEE Trans. Neural Netw. 5, 594-603 (1994)

2. Joya, G, Atencia, M, Sandoval, F: Hopfield neural network for optimization: study of different dynamic. Neurocomputing 43, 219-237 (2002)

3. Cao, J, Zhou, D: Stability analysis of delayed cellular neural networks. Neural Netw. 11, 1601-1605 (1998)

4. Liu, X, Teo, K, Xu, B: Exponential stability of impulsive high-order Hopfield-type neural networks with time-varying delays. IEEE Trans. Neural Netw. 16, 1329-1339(2005)

5. Cao, J, Liang, J: Boundedness and stability for Cohen-Grossberg neural network with time-varying delays. J. Math. Anal. Appl. 296, 665-685 (2004)

6. Arik, S: Global asymptotic stability analysis of bidirectional associative memory neural networks with time delays. IEEE Trans. Neural Netw. 16, 580-586 (2005)

7. Park, J, Park, C, Kwon, O, Lee, S: A new stability criterion for bidirectional associative memory neural networks of neutral-type. Appl. Math. Comput. 199, 716-722 (2008)

8. He, Y, Wang, Q, Wu, M, Lin, C: Delay-dependent state estimation for delayed neural networks. IEEE Trans. Neural Netw. $17,1077-1081(2006)$

9. Huang, H, Feng, G, Cao, J: An LMI approach to delay-dependent state estimation for delayed neural networks. Neurocomputing 71, 2857-2867 (2008)

10. Huang, H, Feng, G: State estimation of recurrent neural networks with time-varying delay: a novel delay partition approach. Neurocomputing 74, 792-796 (2011)

11. Zhang, D, Yu, L: Exponential state estimation for Markovian jumping neural networks with time-varying discrete and distributed delays. Neural Netw. 35, 103-111 (2012)

12. Balasubramaniam, $P$, Vembarasan, $V$, Rakkiyappan, R: Delay-dependent robust exponential state estimation of Markovian jumping fuzzy Hopfield neural networks with mixed random time-varying delays. Commun. Nonlinear Sci. Numer. Simul. 16, 2109-2129 (2011)

13. Balasubramaniam, $\mathrm{P}$, Kalpana, M, Rakkiyappan, R: State estimation for fuzzy cellular neural networks with time delay in the leakage term, discrete and unbounded distributed delays. Comput. Math. Appl. 62, 3959-3972 (2011) 
14. Liu, Y, Wang, Z, Liu, X: State estimation for discrete-time Markovian jumping neural networks with mixed mode-dependent delays. Phys. Lett. A 372, 7147-7155 (2008)

15. Mou, S, Gao, H, Qiang, W, Fei, Z: State estimation for discrete-time neural networks with time-varying delays. Neurocomputing 72, 643-647 (2008)

16. Park, J, Kwon, O, Lee, S: State estimation for neural networks of neutral-type with interval time-varying delays. Appl. Math. Comput. 203, 217-223 (2008)

17. Park, J, Kwon, O: Further results on state estimation for neural networks of neutral-type with time-varying delay. Appl. Math. Comput. 208, 69-75 (2009)

18. Park, J, Kwon, O: Design of state estimator for neural networks of neutral-type. Appl. Math. Comput. 202, 360-369 (2008)

19. Lakshmanan, S, Park, J, Jung, H: State estimation for neural neutral-type networks with mixed time-varying delays and Markovian jumping parameters. Chin. Phys. B 21(10), 100205 (2012)

20. Hu, J, Li, N, Liu, X, Zhang, G: Sampled-data state estimation for delayed neural networks with Markovian jumping parameters. Nonlinear Dyn. 73, 275-284 (2013)

21. Rakkiyappan, R, Sakthivel, N, Park, J, Kwon, O: Sampled-data state estimation for Markovian jumping fuzzy cellular neural networks with mode-dependent probabilistic time-varying delays. Appl. Math. Comput. 221, 741-769 (2013)

22. Lakshmanan, S, Park, J, Rakkiyappan, R, Jung, H: State estimator for neural networks with sampled data using discontinuous Lyapunov functional approach. Nonlinear Dyn. 73, 509-520 (2013)

23. Rakkiyappan, R, Zhu, Q, Radhika, T: Design of sampled data state estimator for Markovian jumping neural networks with leakage time-varying delays and discontinuous Lyapunov functional approach. Nonlinear Dyn. 73, 1367-1383 (2013)

10.1186/1687-1847-2014-138

Cite this article as: Yang et al.: Sampled-data state estimation for neural networks of neutral type. Advances in Difference Equations 2014, 2014:138

\section{Submit your manuscript to a SpringerOpen ${ }^{\circ}$ journal and benefit from:}

- Convenient online submission

- Rigorous peer review

- Immediate publication on acceptance

- Open access: articles freely available online

- High visibility within the field

- Retaining the copyright to your article 